Running head: ATTACHMENT IN-HOUSE CLINICAL CONUNDRUMS

\title{
It Takes A Village: A Call for Engaging Attachment with Adjunct Disciplines to Clarify "In-House" Clinical Conundrums
}

Or Dagan \& Kristin Bernard

Department of Psychology, Stony Brook University, Stony Brook, NY

*Paper accepted for publication at Attachment \& Human Development (ID \# RAHD-2020-SE05 R1); 1/19/21

Reference: Dagan, O. \& Bernard, K. (in press). It Takes A Village: A Call for Engaging Attachment with Adjunct Disciplines to Clarify "In-House" Clinical Conundrums. Attachment \& Human Development.

Correspondent author:

Or Dagan, Ph.D.

Department of Psychology, Stony Brook University

Stony Brook, NY, 11794

or.dagan@stonybrook.edu

+1- 646-270-1100 


\begin{abstract}
Researchers have long discussed the rich clinical applications of attachment theory. Specifically, clinicians have been compelled by the idea that insecure attachment may bring about stressful (real or perceived) interpersonal experiences that increases risk for internalizing symptoms. However, recent meta-analyses examining the links between attachment representations and internalizing symptoms have challenged the assumption that insecure attachment, broadly speaking, is associated with negative mental health outcomes. Rather, findings highlight the importance of considering insecure attachment subtypes in understanding one's vulnerability for internalizing symptoms. Here we expand on this special issue's target papers and propose that, despite an increase in citation impact of clinically relevant attachment research, there are still core theoretical and methodological questions left unanswered. We highlight three clinical conundrums: (1) hyperactivating, but not deactivating, attachment is linked to increased internalizing symptoms in adolescence and adulthood; (2) the magnitude of the associations between insecure attachment subtypes and internalizing symptoms varies depending on the developmental period; and (3) self-reported, but not narrative-based, deactivating attachment is associated with increased internalizing symptoms. We call for engagement with adjunct academic disciplines to elucidate these issues. These clinical conundrums have important ethical implications regarding how we understand insecure attachment and necessitate close theoretical and empirical attention before attachment findings can truly inform clinical practice.
\end{abstract} Keywords: Clinical, insecure attachment, internalizing symptoms 


\section{It Takes A Village: A Call for Engaging Attachment with Adjunct Disciplines to Clarify "In-House" Clinical Conundrums}

Since its development, attachment theorists have considered attachment theory clinically useful. This makes sense, given that attachment processes involve an affective-cognitivebehavioral network that is geared toward reducing distress by achieving proximity to close others. Indeed, findings stemming from attachment theory are often discussed in terms of their clinical implications and applications.

Schuengel et al. (this issue) demonstrate that interest in the clinical implications of attachment theory (i.e., attachment-related interventions, mental health outcomes) is gradually increasing, as evident by a surge in citations of meta-analytic findings on these topics. At the same time, Duschinsky et al.'s (this issue) sociological account of the attachment discourse reveals significant differences in the meaning of attachment-related terms across research and practice contexts. Taken together the findings put forward by Schuengel et al. and Duschinsky et al. reveals a challenge for effective dissemination of health-care: Attachment knowledge is increasingly applied to solve real-life problems (i.e., mental health issues), but attachment discourse is not consolidated "enough" to be safely translated between academic disciplines (e.g., from developmental science to psychotherapy research), and ultimately from academia to clinical use.

In this short commentary, we take a clinical perspective to expand on this special issue's target papers in two ways. First, we propose that, despite an increase in citation impact of clinically relevant attachment research (Schuengel et al., this issue), there are still core theoretical and methodological questions left unanswered. These unanswered questions may be, in part, due to discrepancies in attachment constructs across developmental stages and research 
traditions (Duschinsky et al., this issue). Second, we suggest that core unsettled theoretical and methodological issues may add to the difficulty in translating attachment research into practice. These knowledge gaps limit the clinical utility of attachment theory and should be addressed via engagement with adjunct academic disciplines.

If insights from attachment research are to be channeled toward bettering the wellbeing of individuals and their families, then attachment scholars need to be first clear among themselves regarding the conclusions stemming from the empirical findings. Only then can attachment be responsibly disseminated within clinical practice. In other words, we first need to do some "in-house cleaning" before trying to relay any unified message to clinical practitioners and the public regarding what attachment can tell us about mental health and well-being.

\section{An Umbrella Issue: Do All Bad Things Go Together?}

In almost all academic domains outside of the developmental psychology school of attachment research, and certainly in the discourse of non-academic clinicians, insecure attachment representations are generally perceived as either a causal factor or a correlate of psychopathological symptoms (Duschinsky et al., this issue). However, research from the past four decades does not allow us to theoretically, empirically, and ethically justify such claims, at least not when it comes to internalizing symptoms. Specifically, two meta-analyses examined the link between attachment representations (as derived from the Adult Attachment Interview [AAI; Main et al., 2003-2008]) and internalizing symptoms. Both concluded that insecure-preoccupied (hereafter, hyperactivating), but not insecure-dismissing (hereafter, deactivating) individuals, endorsed elevated depressive and anxiety symptoms relative to their securely attached counterparts (Dagan et al., 2018, 2020). These results raise questions about the significance of insecure attachment representations in the development and maintenance of internalizing 
symptoms, suggesting that not all bad things (i.e., subtypes of insecure attachment representations and internalizing symptoms) go together.

The importance of differentiating insecure attachment subtypes when predicting developmental outcomes is by no means new. Building upon previous insights (e.g., Erickson et al., 1985; see also Sroufe, this issue), we highlight three unsettled issues about the role of insecure attachment in the emergence and maintenance of internalizing symptoms ${ }^{1}$. First, we discuss our limited understanding of the mechanisms explaining the differential mental health outcomes associated with each insecure attachment representation subtype (see Issue 1 below). Second, we explore differences in meta-analytic associations between insecure attachment representation subtypes and internalizing symptoms in adolescence and adulthood versus those between insecure attachment pattern subtypes (as assessed via the Strange Situation Procedure; SSP [Ainsworth et al., 1978]) and internalizing symptoms in childhood (see Issue 2 below). Third, we consider that the pattern of associations between insecure attachment subtypes and internalizing symptoms differ based on how attachment is assessed (i.e., AAI following the developmental psychology tradition vs. self-report following the social psychology tradition; see Issue 3 below). Echoing Schuengel et al. (this issue), we argue that moving attachment research to the level of engagement- in this case, engagement with adjunct academic disciplines- is essential in helping the attachment field better understand these "in-house" issues.

\footnotetext{
${ }^{1}$ Of note, given the limited space, in this commentary we do not elaborate on attachment disorganization; for an in-depth discussion on this attachment pattern, see Haltigan et al. (this issue).
} 
For each unsettled issue, we describe the 'conundrum' in terms of either non-theoretically consistent or inconsistent empirical results; discuss how engaging with adjunct research disciplines may help generate new hypotheses; and highlight potential explanatory (and in one case, ethical) outcomes of such engagement. Of note, we acknowledge that efforts to engage with related academic disciplines have been taking place. Our hope is to define these engagement efforts in a framework that addresses unresolved clinical issues to encourage more close and routine engagement with these disciplines.

Before moving forward, a crucial point should be explicitly made. Duschinsky et al. (this issue) cautioned against academic discourse "shorthands," and encouraged specifying the meaning of attachment-related terms for the sake of unifying attachment discourse across disciplines. With that in mind, we use here the terms "hyperactivating" and "deactivating" to denote the tendency to exhibit heightened (in hyperactivating) or reduced (in deactivating) proximity seeking behaviors at times of distress (for an excellent discussion on how language can be understood as an attachment behavior that signals proximity needs in the context of psychotherapy, see Talia et al., 2014). We reason that such definitions denote the core feature of the attachment behavioral system, and can be operationalized using observational (e.g., signaling and physically moving toward or away from caregivers during the SSP) and interview-based (e.g., verbally engaging with or disengaging from memories of caregivers) attachment measures across the lifespan. Relatedly, research on the taxonicity and factor structure of individual differences in attachment as assessed in infancy (with the SSP) and in adolescence/adulthood (with the AAI) have provided evidence that the variation in attachment during these developmental stages is well captured by two weakly correlated dimensions of deactivation and hyperactivation (Fraley \& Spieker, 2003; Groh et al., 2019; Haltigan et al., 2014; Raby et al., 
2020). Such evidence lends empirical support to the conceptualization of attachment across the lifespan in the manner we propose here.

\section{Issue 1: Depending on The Subtype, Insecure Attachment Representation May or May Not Be Associated with Internalizing Symptoms}

The Conundrum. Two recent meta-analyses examined the link between attachment representations in post-childhood years and internalizing symptoms of depression and anxiety (Dagan et al., 2018, 2020). The results challenged the conventional view that is commonly portrayed in popular media (see Issue 2 below) and attachment research using self-report (see Issue 3 below); that is, that an insecure attachment representation, regardless of its subtype, increases risk for internalizing symptoms. Specifically, hyperactivating individuals (who tend to approach others excessively when in distress) reported significantly more internalizing symptoms than deactivating individuals (who tend to withdraw from others when in distress) and securely attached individuals (who tend to flexibly move between the two approach-withdrawal polarities).

Adjunct Discipline to Engage With: Clinical Psychological Science. Despite a vast literature that derives clinical implications from attachment theory (e.g., Atkinson \& Goldberg, 2003; Holmes \& Slade, 2018; Rutter, 1995; Zeanah et al., 2011), there has been little effort to integrate clinical psychological theories and methodologies with attachment theory to explain attachment phenomena. However, attachment theory and research are well positioned to integrate clinical psychological science, given that both disciplines identify cognitive-affective bases for psychopathological phenomena. Cognitive-affective science may help explain the mechanisms underlying the recent meta-analytic findings on the divergent links between insecure attachment representations and internalizing symptoms. 
As summarized by Duschinsky (2020), Mary Main framed attachment behavior, including its discourse manifestations during the AAI, as reflecting attentional processes. Specifically, insecurely attached individuals tend to exhibit discourse that reflects either a diversion of attention away from attachment experiences with their caregivers (as in the case of deactivating individuals), or an inability to emotionally distance oneself from these experiences enough to evaluate and reflect on them (as in the case of hyperactivating individuals).

In clinical psychological science, attentional processes are central to understanding psychopathology (Shechner et al., 2012). A central construct for understanding the development and maintenance of fear and distress- two key vulnerability factors in internalizing disorders- has been threat-related attentional bias (Bar-Haim et al., 2007). Threat-related attentional bias refers to the enhanced tendency to attend to, process, and remember threatening stimuli (Mogg \& Bradley, 1998). Biased processing of threatening interpersonal information may lead to a persistent interpretation of others' behavior as hostile or untrustworthy; this, in turn, may lead to perceived social isolation and lack of support, which have been consistently linked to internalizing symptoms in adolescence and adulthood (Rueger et al., 2016; Santini et al., 2015).

Thus, Main's interpretation of attachment behaviors and discourse as attentional processes is aligned with empirically-based mechanisms of psychopathology. Returning to our first conundrum, threat-related attentional bias may explain why AAI discourse of hyperactivating, but not deactivating, individuals entails higher susceptibility to internalizing symptoms. Hyperactivating individuals may be more susceptible to internalizing symptoms because of their heightened threat-related attentional bias, reflected in their excessive engagement with negative past and present memories and experiences with their caregivers. Deactivating individuals may be at lower risk for experiencing internalizing symptoms because 
they have lower threat-related attentional bias, as reflected in their diversion of attention away from potentially negative attachment memories.

Potential Outcomes. According to recent meta-analytic findings, the question of interest has become: Why do hyperactivating individuals report significantly higher internalizing symptoms than deactivating individuals? Clinical psychological science has traditionally dealt with causes for psychopathological symptoms and the mechanisms underlying them and is thus well suited to answer such a question.

Applying clinically informed mechanisms to theory and research on the differential links between insecure attachment representation subtypes and internalizing symptoms is crucial for intervention science. It is possible that deactivating strategies may be adaptive (see more under Issue 2 below). If we reinterpret deactivating individuals' attentional strategy of avoidance as a strategy that alleviates distress, this may shift how we approach case conceptualization and treatment. Additionally, integrating aspects of mechanism-focused clinical interventions (e.g., Attention Bias Modification [Hakamata et al., 2010]), with attachment-based interventions (e.g., Attachment-Based Family Therapy for Depressed Adolescents [Diamond et al., 2002]) and psychotherapy principles (e.g., Holmes, 1996) may prove a more robust and well-rounded therapeutic intervention compared with either one of the approaches alone.

\section{Issue 2: Depending on The Development Period, Subtypes of Insecure Attachment May or May Not Be Associated with Internalizing Symptoms}

The Conundrum. Two large meta-analyses reported that children with histories of deactivating (i.e., insecure-avoidant), but not hyperactivating (i.e., insecure-resistant), attachment patterns in infancy and early childhood showed elevated internalizing symptoms (Groh et al., 2012; Madigan et al., 2013; but see results from the Minnesota Longitudinal Study of Resilience 
and Adaptation, suggesting that hyperactivating, but not deactivating or securely attached children tended to experience anxiety disorders in adolescence; Warren et al., 1997). In contrast, adolescents and adults with hyperactivating, but not deactivating, attachment classifications showed elevated internalizing symptoms, as evidenced in meta-analyses described above (Dagan et al., 2018, 2020). Thus, the nature of the association between subtypes of insecure attachment and mental health may be different across developmental periods (Dagan \& Bernard, 2019).

Of note, we acknowledge that attachment behavior observed in infancy and childhood (in the SSP) is measured differently than attachment discourse that is observed in adolescence and adulthood (in the AAI). However, given our specified definitions of hyperactivating and deactivating attachment, we argue that observed behaviors in the SSP and in the AAI reflect variability in proximity seeking (i.e., in physical movement and verbal behavior during the SSP, and the quality and coherence of speech during the AAI). Such conceptualization, though, is still in need for empirical validation.

Adjunct Discipline to Engage With: Evolutionary Psychology. Bowlby integrated evolutionary themes to inform attachment theory. Main (1979) followed this path by conceptualizing insecure attachment as adaptations, or "second best" conditional strategies, to one's caregiving environment that serves to optimize proximity to caregivers when they are unavailable or responding inconsistently to distress cues. Accordingly, there has been an assumption that disturbances in early relationships with caregivers, often reflected in insecure attachment patterns as measured via the SSP, are risk factors for psychopathology, including internalizing symptoms (Egeland \& Carlson, 2004). However, considering recent meta-analytic findings, such clinical viewpoint is too narrow as it lacks the ability to explain the differential 
vulnerability to internalizing symptoms between insecure attachment pattern subtypes (in childhood) and representation subtypes (post-childhood), and across developmental periods.

When attempting to understand the discrepant links between insecure attachment subtypes and internalizing symptoms across the lifespan, attachment theory and research may turn to the Evolutionary Mismatch Hypothesis. This hypothesis is defined as the "adaptive lag that occurs if the environment that existed when a mechanism evolved changes more rapidly than the time needed for the mechanism to adapt to the change" (Li et al., 2018, p. 38). Multiple evolutionary-developmental models of health and disease (e.g., Frankenhuis \& Del Giudice, 2012) suggest that health problems result from a mismatch between the affective, cognitive, and behavioral adaptations one develops in early environment and the fit of those adaptations in the environment one inhabits as an adult.

The mismatch hypothesis predicts that a move from a stressful to a non-stressful environment (or vice versa) may lead to a mismatch between one's early developmental adaptive pattern of insecure attachment behavior and the social demands of the specific developmental period. In short, there are two trajectories that may be predicted by the mismatch hypothesis. The first is that children who develop responses that are geared towards reducing stress via detection and monitoring of danger may find that their once adaptive strategies are no longer 'useful' in a later, non-stressful social environment that does not call for such adaptation. The opposite predicted developmental trajectory is that children who grow up in safe and supportive environments develop and consolidate their social strategies in a manner that may not 'work' if used in later stressful environments.

A social environment may be stressful- and hence as one that increases risk for internalizing symptoms- depending on the degree to which each insecure attachment pattern (in 
childhood) or representation (in adolescence and adulthood) matches with the developmentallyappropriate interpersonal support expectations. Given the transition from an environment in which emotional support is almost entirely expected from parents (i.e., childhood) to one in which such support is expected primarily from peers and romantic partners (i.e., adolescence and adulthood), the evolutionary mismatch hypothesis will predict the following. First, hyperactivating individuals will experience increased distress as they move from childhood to post-childhood developmental stages. Whereas their orientation towards soliciting support from caregivers during childhood (via execution of excessive cues for help) is developmentally appropriate in childhood, this orientation may no longer achieve its goal in a non-parental social environment. In adolescents and adulthood, hyperactivating individuals will experience a mismatch between their attachment pattern, which entails excessive dependency on parental support, and the appropriate developmental milestone of autonomy from caregivers; this mismatch will increase their vulnerability to internalizing symptoms. The second prediction is that deactivating individuals will experience a decrease in distress when transitioning from childhood to post-childhood developmental stages. Whereas the belief in parental unavailability at times of need may create heightened distress during childhood years, by adolescence, deactivating individuals may be well accustomed to deal with stressors by themselves. This selfreliant psychological capacity, in turn, matches the post-childhood social demand of autonomy from caregivers, thereby decreasing the vulnerability to internalizing symptoms.

Potential Outcomes. Engaging with evolutionary theoretical models and research paradigms may help attachment research in two major ways. First, it may generate a framework that supports an understanding of how the same strategies may yield different vulnerabilities to internalizing symptoms in different developmental stages. Such a framework can propel novel 
testable hypotheses regarding the adaptivity of insecure attachment subtypes across the lifespan. Integrating insights from recent evolutionary hypotheses, such as the mismatch hypothesis, may thus open new avenues for research that may not only explain existing meta-analytic findings, but also expand attachment theory accordingly.

Above and beyond the explanatory power of evolutionary theories, there are also significant ethical implications for highlighting the adaptive value of insecure attachment patterns across the lifespan. In popular media, individual differences in attachment patterns are commonly portrayed as 'good' and 'bad' personality characteristics. The idea of stripping away a relational pattern that serves an ontogenetic evolutionary purpose (i.e., to lessen distress via ensuring optimal proximity to caregivers or attenuate further painful rejection) and assigning it back to the person as a personality characteristic (e.g., socially distant or has 'difficulty' in forming close relationships) is not only misinformed but also entails questionable ethical claims. Whereas secure attachment is "good" and should be the gold standard for healthy life, insecure attachment across the lifespan is "bad" and should be "treated" not only for the sake of the insecure individuals but also for the sake of both the individual's close others and greater society. Examples are numerous, running the gamut from identifying insecure individuals as 'having no feelings' or being 'erratic and unpredictable' (Kassel, 2020), to identifying them as threats to the well-being of society (by being non-compliant with the societal health demands of wearing face masks during COVID-19 pandemic [Narvaez, 2020]). Engaging with the mismatch hypothesis when discussing insecure attachment subtypes across the lifespan can lessen the degree to which attachment insecurity is perceived as "abnormal" or "deviant." This shift in interpretation highlights potential reasons behind the mental health struggles of some individuals with insecure 
attachment, which is critical for fostering an empathic stance towards them. In itself, such an empathic stance can be strongly therapeutic.

\section{Issue 3: Depending on the Attachment Assessment Tradition, Insecure Attachment} Subtype May or May Not Be Associated with Internalizing Symptoms

The Conundrum. Depending on the methodological tradition, deactivating individuals may or may not be at elevated risk for internalizing symptoms. A large body of research using a self-report attachment questionnaire indicates that individuals who score higher on the deactivating dimension tend to endorse more internalizing symptoms than individuals who score lower on this dimension (e.g., Mikulincer \& Shaver, 2012). Contrary to these findings, two recent meta-analyses (Dagan et al., 2018, 2020) established that AAI classified deactivating individuals did not endorse higher internalizing symptomatology than non-deactivating individuals. Moreover, the only study that compared links between attachment as assessed using self-report and attachment as assessed using the AAI and internalizing symptoms (Fortuna \& Roisman, 2008) reported that more internalizing symptoms were associated with higher scores of the self-reported deactivating dimension, but with lower scores on the AAI deactivating dimension.

Adjunct Discipline to Engage With: Social Psychology. Whereas the social-personality tradition has used self-reports to assess post-childhood attachment 'styles,' the developmental tradition has heavily relied on a semi-structured interview (most commonly, AAI) to assess postchildhood attachment representations. A line of research led by Roisman (2009) highlighted that, despite common conceptual roots, these two schools of adult attachment research have developed in parallel to each other, resulting in adult attachment measures that are only trivially linked to one another (Roisman et al., 2007). Specifically, the correlation between the two 
indicators of deactivating attachment (i.e., self-reported avoidant attachment style and dismissing attachment pattern as assessed via the AAI) has a mean weighted effect size near zero. Despite Roisman's findings that emerged over a decade ago, little has changed in the conceptual and methodological understanding of the reasons for the marginal associations between the two attachment tradition assessments. This is reflected in the divergent findings from the two adult attachment traditions as to the associations between adult deactivating attachment and internalizing symptoms.

The empirical rift between the attachment traditions highlighted by Roisman and colleagues- which has a long history (Duschinky, 2020, pp. 427-536)- is now acknowledged by attachment researchers. However, limited attempts have been made to explore the divergent findings in terms of clinical research. Multiple points of difference between the two attachment traditions' assessments have been proposed (e.g., Bartholomew \& Shaver 1998). However, neither assessment tradition has yet to initiate a programmatic body of research towards understanding in what context and why do the two adult attachment assessments differ, and perhaps complement each other. This is unfortunate. From the point of view of the developmental tradition, clinical applications of attachment can greatly benefit from incorporating experimental causality testing that is often utilized in social psychological science to clarify the temporal links between attachment patterns and internalizing symptoms.

Potential Outcomes. Schuengel et al. (this issue) noted that, unlike the literature on attachment representations, which is most often assessed via the AAI, literature on self-report attachment styles in adolescence and adulthood has surged. In the near future, the surge in selfreport, but not AAI attachment research may create an imbalance in the clinical literature. Such imbalance may lead to the adoption of uniformed literature by clinicians who may use it when 
applying attachment-based interventions for internalizing symptoms. However, such practice, as we argue here, may be rather premature.

Theoretically, attachment theory claims that insecure attachment tends to lead to heightened vulnerability to internalizing symptoms through expectations of caregiving unavailability under distress (Bowlby, 1973, 1980; Sroufe et al., 2005). However, as it stands now, the different attachment assessment traditions are at odds with respect to the veracity of such claim, at least with regards to deactivating individuals and internalizing symptoms. Each attachment tradition can provide a sound explanation as to the role that deactivating attachment plays in vulnerability to internalizing symptoms. But, when it ultimately comes to clinical practice, such disagreements (versus a clearer evidence-based approach) may be harmful.

Clarifying the conundrum at hand can greatly inform potential modifications of attachment theory and practice. Moving towards a better understanding of which attachment measure is more useful in conceptualizing vulnerability to internalizing symptoms postchildhood can be achieved in various ways. One approach would be to longitudinally assess the predictive validity of both attachment measurements in explaining variability in internalizing symptoms within the same sample. Ideally, such a study would include a comprehensive assessment battery for internalizing symptoms, including measurement of biomarkers (Peterson \& Weissman, 2011) and multiple informant reports (De Los Reyes et al., 2012). A multi-method approach may also help reveal whether these attachment assessments complement each other in arriving at a more holistic understanding of individual differences in internalizing symptoms.

Clinically, if deactivating attachment is positively associated with internalizing symptoms (as indicated in the self-report literature), deactivating attachment strategies may be considered maladaptive, and interventions should focus on adjusting these strategies to alleviate 
internalizing symptoms. However, if deactivating attachment is associated with little to no internalizing symptomatology (as indicated in AAI literature), one may deem it adaptive, and clinically support elements of this strategy when intervening with individuals who may suffer from internalizing symptoms associated with other sources of distress.

\section{Conclusion}

In line with one of Bowlby's main aims, attachment theory and research clearly hold clinical implications for therapeutic interventions. Schuengel et al. (this issue) present evidence of an increase in clinical applications of attachment theory in recent years. Moreover, Duschinsky et al. (this issue) caution that we may 'talk over each other' when we communicate such empirical evidence amongst ourselves and with practitioners and the public. Resolving the "in-house" clinical conundrums we highlight here may inform attachment theory and practice, and propel a more cohesive attachment discourse. For the time being, attachment theory is yet to be "light enough to travel" from academia to clinical practice.

The responsibility for responding to the call for "in house" cleaning falls on attachment researchers. Accordingly, moving attachment to the level of engagement necessitates active efforts to collaborate with researchers from multidisciplinary fields, invite their methodological expertise, and incorporate their perspectives in developmental research on attachment across the lifespan. Such engagement can promote novel and testable hypotheses that may expand both attachment theory and its applicability- yielding a more comprehensive understanding of the intricate dynamics between insecure attachment and internalizing symptoms across the lifespan. 


\section{References}

Ainsworth, M. D. S., Blehar, M. C., Waters, E., \& Wall, S. N. (1978). Patterns of attachment. Hillsdale, NJ: Erlbaum.

Atkinson, Leslie, \& Goldberg, S. (2003). Attachment issues in psychopathology and intervention. Routledge.

Bar-Haim, Y., Lamy, D., Pergamin, L., Bakermans-Kranenburg, M. J., \& van IJzendoorn, M. H. (2007). Threat-related attentional bias in anxious and nonanxious individuals: A metaanalytic study. Psychological Bulletin, 133(1), 1-24. https://doi.org/10.1037/00332909.133.1.1

Bartholomew, K. \& Shaver, P.R. (1998) Methods of assessing adult attachment: Do they converge? In J.A. Simpson \& W.S. Rholes (eds), Attachment Theory and Close Relationships (pp.25-45). New York: Guilford.

Bowlby, J. (1973). Attachment and Loss: Vol. 2. Separation: Anxiety and anger. New York, NY: Basic Books.

Bowlby, J. (1980). Attachment and Loss: Vol. 3. Loss: Sadness and depression. New York: Basic Books.

Dagan, O., \& Bernard, K. (2019, September 30). The paradox of insecure attachment and depressive symptoms across the lifespan: Towards an integrative view. doi:10.31219/ osf.io/jbsth

Dagan, O., Facompré, C. R., Nivison, M. D., Roisman, G. I., \& Bernard, K. (2020). Preoccupied and dismissing attachment representations are differentially associated with anxiety in adolescence and adulthood: A meta-analysis. Clinical Psychological Science, 8(4), 614640. https://doi.org/10.1177/2167702620917455

Dagan, O, Facompré, C. R., \& Bernard, K. (2018). Adult attachment representations and 
depressive symptoms: A meta-analysis. Journal of Affective Disorders, 236, 274-290. https://doi.org/https://doi.org/10.1016/j.jad.2018.04.091

De Los Reyes, A., Thomas, S. A., Goodman, K. L., \& Kundey, S. M. A. (2013). Principles underlying the use of multiple informants' reports. Annual Review of Clinical Psychology, 9(1), 123-149. https://doi.org/10.1146/annurev-clinpsy-050212-185617

Diamond, G. S., Reis, B. F., Diamond, G. M., Siqueland, L., \& Isaacs, L. (2002). Attachmentbased family therapy for depressed adolescents: A treatment development study. Journal of the American Academy of Child \& Adolescent Psychiatry, 41(10), 1190-1196.

Duschinsky, R. (2020). Cornerstones of attachment research. Oxford: Oxford University Press. Egeland, B., \& Carlson, E. A. (2004). Attachment and psychopathology. In L. Atkinson \& S. Goldberg (Eds.), Attachment issues in psychopathology and intervention (pp. 27-48). Mahwah, NJ, US: Lawrence Erlbaum Associates Publishers.

Erickson, M. F., Sroufe, L. A., \& Egeland, B. (1985). The relationship between quality of attachment and behavior problems in preschool in a high-risk sample. Monographs of the Society for Research in Child Development, 50(1/2), 147-166. https://doi.org/10.2307/3333831

Fortuna, K., \& Roisman, G. I. (2008). Insecurity, stress, and symptoms of psychopathology: Contrasting results from self-reports versus interviews of adult attachment. Attachment and Human Development, 10(1), 11-28. https://doi.org/10.1080/14616730701868571

Fraley, R. C., \& Spieker, S. J. (2003). Are infant attachment patterns continuously or categorically distributed? A taxometric analysis of strange situation behavior. Developmental Psychology, 39(3), 387-404. https://doi.org/10.1037/0012-1649.39.3.387

Frankenhuis, W. E., \& Del Giudice, M. (2012). When do adaptive developmental mechanisms 
yield maladaptive outcomes? Developmental Psychology, 48(3), 628-642.

\section{https://doi.org/10.1037/a0025629}

Groh, A. M., \& Narayan, A. J. (2019). Infant attachment insecurity and baseline physiological activity and physiological reactivity to interpersonal stress: A meta-analytic review. Child Development, 90(3), 679-693. https://doi.org/10.1111/cdev.13205

Groh, A. M., Roisman, G. I., van IJzendoorn, M. H., Bakermans-Kranenburg, M. J., \& Fearon, R. P. (2012). The significance of insecure and disorganized attachment for children's internalizing symptoms: A meta-analytic study. Child Development, 83(2), 591-610. https://doi.org/10.1111/j.1467-8624.2011.01711.x

Hakamata, Y., Lissek, S., Bar-Haim, Y., Britton, J. C., Fox, N. A., Leibenluft, E., Ernst, M., \& Pine, D. S. (2010). Attention Bias Modification Treatment: A Meta-Analysis Toward the Establishment of Novel Treatment for Anxiety. Biological Psychiatry, 68(11), 982-990. https://doi.org/https://doi.org/10.1016/j.biopsych.2010.07.021

Haltigan, J. D., Roisman, G. I., \& Haydon, K. C. (2014). II. The latent structure of the adult attachment interview: Exploratory and confirmatory evidence. Monographs of the Society for Research in Child Development, 79(3), 15-35. https://doi.org/10.1111/mono.12111

Holmes, J. (1996). Attachment, intimacy and autonomy: Using attachment theory in adult psychotherapy. Northdale, NJ: Jason Aronson.

Holmes, J., \& Slade, A. (2018). Attachment in therapeutic practice. London, UK: Routledge.

Kassel, G. (2020). Attachment Theory Plays a Role in Relationships - Here's What That Means for You. https://www.healthline.com/health/relationships/attachment-theory

Li, N. P., van Vugt, M., \& Colarelli, S. M. (2018). The evolutionary mismatch hypothesis: Implications for psychological Science. Current Directions in Psychological Science, 27(1), 
38-44. https://doi.org/10.1177/0963721417731378

Madigan, S., Atkinson, L., Laurin, K., \& Benoit, D. (2013). Attachment and internalizing behavior in early childhood: A meta-analysis. Developmental Psychology, 49(4), 672-689. https://doi.org/10.1037/a0028793

Main, M. (1979). The ultimate causation of some infant attachment phenomena: Further answers, further phenomena, and further questions. Behavioral and Brain Sciences, 2(4), 640-643. https://doi.org/DOI: 10.1017/S0140525X00064992

Main, M., Goldwyn, R., \& Hesse, E. (2003-2008). Adult attachment scoring and classification system. Unpublished manuscript, University of California at Berkeley.

Mikulincer, M., \& Shaver, P. R. (2012). An attachment perspective on psychopathology. World Psychiatry, 11(1), 11-15. https://doi.org/10.1037/14498-005

Mogg, K., \& Bradley, B. P. (1998). A cognitive-motivational analysis of anxiety. Behaviour Research and Therapy, 36(9), 809-848. https://doi.org/https://doi.org/10.1016/S00057967(98)00063-1

Narvaez, D. F. (2020). Why Are Some People Noncompliant With Stay-at-Home Orders? Avoidant attachment may drive noncompliance. https://www-psychologytodaycom.cdn.ampproject.org/c/s/www.psychologytoday.com/us/blog/morallandscapes/202005/why-are-some-people-noncompliant-stay-home-orders?amp\#

Peterson, B. S., \& Weissman, M. M. (2011). A brain-based endophenotype for Major Depressive Disorder. Annual Review of Medicine, 62(1), 461-474. https://doi.org/10.1146/annurev$\underline{\text { med-010510-095632 }}$

Raby, K. L., Verhage, M. L., Fearon, R. M. P., Fraley, R. C., Roisman, G. I., van IJzendoorn, M. H., Schuengel, C., Madigan, S., Oosterman, M., Bakermans-Kranenburg, M. J., Bernier, A., 
Ensink, K., Hautamäki, A., Mangelsdorf, S., Priddis, L. E., \& Wong, M. S. (2020). The latent structure of the adult attachment interview: Large sample evidence from the collaboration on attachment transmission synthesis. Development and Psychopathology, 113. https://doi.org/DOI: 10.1017/S0954579420000978

Roisman, G. I. (2009). Adult attachment: Toward a rapprochement of methodological cultures. Current Directions in Psychological Science, 18(2), 122-126. https://doi.org/10.1111/j.1467-8721.2009.01621.x

Roisman, G. I., Holland, A., Fortuna, K., Fraley, R. C., Clausell, E., \& Clarke, A. (2007). The Adult Attachment Interview and self-reports of attachment style: An empirical rapprochement. Journal of Personality and Social Psychology, 92(4), 678-697. https://doi.org/10.1037/0022-3514.92.4.678

Rueger, S. Y., Malecki, C. K., Pyun, Y., Aycock, C., \& Coyle, S. (2016). A meta-analytic review of the association between perceived social support and depression in childhood and adolescence. Psychological Bulletin, 142(10), 1017-1067. https://doi.org/10.1037/bul0000058

Rutter, M. (1995). Clinical implications of attachment concepts: Retrospect and prospect. Journal of Child Psychology and Psychiatry, 36(4), 549-571. https://doi.org/10.1111/j.1469-7610.1995.tb02314.x

Santini, Z. I., Koyanagi, A., Tyrovolas, S., Mason, C., \& Haro, J. M. (2015). The association between social relationships and depression: A systematic review. Journal of Affective Disorders, 175, 53-65. https://doi.org/https://doi.org/10.1016/j.jad.2014.12.049

Shechner, T., Britton, J. C., Pérez-Edgar, K., Bar-Haim, Y., Ernst, M., Fox, N. A., Leibenluft, E., \& Pine, D. S. (2012). Attention biases, anxiety, and development: Toward or away from 
threats or rewards? Depression and Anxiety, 29(4), 282-294.

\section{https://doi.org/10.1002/da.20914}

Sroufe, L. A., Egeland, B., Carlson, E., \& Collins, W. A. (2005). The development of the person: The Minnesota study of risk and adaptation from birth to adulthood. New York: Guilford.

Talia, A., Daniel, S. I. F., Miller-Bottome, M., Brambilla, D., Miccoli, D., Safran, J. D., \& Lingiardi, V. (2014). AAI predicts patients' in-session interpersonal behavior and discourse: A "move to the level of the relation" for attachment-informed psychotherapy research. Attachment and Human Development, 16(2), 192-209.

https://doi.org/10.1080/14616734.2013.859161

Warren, S., Huston, L., Egeland, B., \& Sroufe, L. A. (1997). Child and adolescent anxiety disorders and early attachment. Journal of the American Academy of Child and Adolescent Psychiatry, 36, 637-644.

Young, K., Sandman, C., \& Craske, M. (2019). Positive and negative emotion regulation in adolescence: Links to anxiety and depression. Brain Sciences, 9(4), 76.

\section{https://doi.org/10.3390/brainsci9040076}

Zeanah, C.H., Berlin, L.J. and Boris, N.W. (2011), Practitioner Review: Clinical applications of attachment theory and research for infants and young children. Journal of Child Psychology and Psychiatry, 52, 819-833. https://doi.org/10.1111/j.1469-7610.2011.02399.x 
Running head: ATTACHMENT IN-HOUSE CLINICAL CONUNDRUMS

\title{
Table 1
}

Adjunct Research Disciplines to Engage with In Order to Elucidate "In House" Clinical

\author{
Conundrums.
}

\begin{tabular}{|l|c|c|}
\hline THE CONUNDRUM & ADJUNCT DISCIPLINES & EXAMPLE RESEARCH AREA \\
\hline $\begin{array}{l}\text { Divergent links between } \\
\text { insecure attachment subtypes } \\
\text { and internalizing symptoms }\end{array}$ & Clinical Psychological Science & Threat-related attentional bias \\
\hline $\begin{array}{l}\text { Divergent links between } \\
\text { insecure attachment subtypes } \\
\text { and internalizing symptoms } \\
\text { across developmental periods }\end{array}$ & Evolutionary Psychology & Evolutionary mismatch hypothesis \\
\hline $\begin{array}{l}\text { Divergent links between } \\
\text { measurement approaches (self- } \\
\text { reported attachment style vs. } \\
\begin{array}{l}\text { AAI attachment patterns) and } \\
\text { internalizing symptoms }\end{array}\end{array}$ & Social Psychology & Romantic attachment styles \\
\hline
\end{tabular}

